Comment. Math. Helv. 75 (2000) 410-414

(C) 2000 Birkhäuser Verlag, Basel

$0010-2571 / 00 / 030410-5 \$ 1.50+0.20 / 0$

Commentarii Mathematici Helvetici

\title{
Universal octonary diagonal forms over some real quadratic fields
}

\author{
Byeong Moon Kim
}

\begin{abstract}
In this paper, we will prove there are infinitely many integers $n$ such that $n^{2}-1$ is square-free and $\mathbb{Q}\left(\sqrt{n^{2}-1}\right)$ admits universal octonary diagonal quadratic forms.
\end{abstract}

Mathematics Subject Classification (2000). Primary 11E12, Secondary 11E20.

Keywords. Universal quadratic forms, real quadratic fields.

\section{Introduction}

A universal integral form over totally real number field $K$ is a positive definite quadratic form over the ring of integers of $K$ which represents all the totally positive integers of $K$. For example, the sum of four squares is universal integral over $\mathbb{Q}$. In 1917 , Ramanujan [8] found there are exactly 54 universal positive diagonal integral quadratic forms over $\mathbb{Q}$. More concretely, he showed there are 54 diagonal quaternary quadratic forms $f(x, y, z, w)=a x^{2}+b y^{2}+c z^{2}+d w^{2}$ such that $a, b, c, d \in \mathbb{Z}^{+}$and the equation $f=n$ is solvable for all $n \in \mathbb{Z}^{+}$. In 1947, M. Willerding [10] proved there are exactly 178 classic universal integral forms. More concretely, she showed there are 178 quaternary quadratic forms $f(x, y, z, w)$ up to equivalence such that $f$ is positive definite integral quadratic form, the coefficients of cross terms of $f$ are always even and the equation $f=n$ is solvable for all $n \in \mathbb{Z}^{+}$. On the other hand, the study of positive universal quadratic integral forms over totally real number fields was initiated by F. Götzky [3]. In 1928, he proved that the sum of four squares is universal over $\mathbb{Q}(\sqrt{5})$. H. Maass $[6]$ improved this result. In 1941, he proved the sum of three squares is positive universal over $\mathbb{Q}(\sqrt{5})$. Four years later, Siegel $[9]$ proved $\mathbb{Q}(\sqrt{5})$ is the only totally real number field, other than $\mathbb{Q}$, over which every (totally) positive integer is a sum of squares. In other words, he showed if a totally real number field $K$ is different from $\mathbb{Q}$ and $\mathbb{Q}(\sqrt{5})$, there is a totally positive algebraic integer $\alpha$ of $\mathrm{K}$ which cannot be represented by the sum of any number of squares. For example, if $K=\mathbb{Q}(\sqrt{2}), \alpha=2+\sqrt{2}$. In 1996, W. K. Chan, M.-H. Kim and S. Raghavan [1] 
classified all (totally) positive universal integral ternary lattices over real quadratic fields. Only $\mathbb{Q}(\sqrt{2}), \mathbb{Q}(\sqrt{3})$ and $\mathbb{Q}(\sqrt{5})$ admit universal integral ternary lattices and total number of universal integral ternary lattices over real quadratic fields is 11. Recently, the author [5] proved there are only finitely many real quadratic fields which admit universal integral septenary diagonal forms. The content of this paper is to prove if $n^{2}-1$ is square-free, there are universal octonary diagonal forms over $\mathbb{Q}\left(\sqrt{n^{2}-1}\right)$. So we can prove there are infinitely many real quadratic fields which admit universal integral octonary diagonal forms. Obviously 8 is the minimal rank with this property.

\section{Main Theorem}

Throughout this chapter, we let $m=n^{2}-1$ be a positive square free integer, $K=\mathbb{Q}(\sqrt{m})$ and $\mathcal{O}_{K}$ be the ring of algebraic integers of $K$. Note that $\epsilon=n+\sqrt{m}$ is the fundamental unit of $\mathcal{O}_{K}$ and is totally positive.

Theorem 1. The octonary diagonal form $x_{1}^{2}+x_{2}^{2}+x_{3}^{2}+x_{4}^{2}+\epsilon x_{5}^{2}+\epsilon x_{6}^{2}+\epsilon x_{7}^{2}+\epsilon x_{8}^{2}$ is universal over $\mathcal{O}_{K}$.

This Theorem is a consequence of following Lemmas.

Lemma 1. Let $1 \leq b<2 n . \alpha=a+b \sqrt{m}$ is totally positive algebraic integer in $K$ if and only if $n b \leq a$.

Proof. As $n b+b \sqrt{m}=b(n+\sqrt{m})$ is totally positive, the necessity is trivial. For the sufficiency, it suffices to prove $n b-1-(b \sqrt{m})<0$. This follows from

$$
\begin{gathered}
(n b-1)^{2}-(b \sqrt{m})^{2}=n^{2} b^{2}-2 n b+1-b^{2}\left(n^{2}-1\right) \\
=(b-n)^{2}-n^{2}+1 \leq(n-1)^{2}-n^{2}+1<0 .
\end{gathered}
$$

Lemma 2. If $\alpha \in \mathcal{O}_{K}^{+}, \alpha$ belongs to

$$
S=\left\{a_{0} \epsilon^{k}+a_{1} \epsilon^{k+1}+\ldots+a_{l} \epsilon^{k+l} \mid k, l \in \mathbb{Z}, a_{0}, a_{1}, \ldots, a_{l} \in \mathbb{N}\right\} .
$$

Proof. Suppose $\alpha=a+b \sqrt{m} \notin S$. We may assume that $b>0$ and $\operatorname{tr}_{K / \mathbb{Q}}(\alpha) \leq$ $\operatorname{tr}_{K / \mathbb{Q}}(\beta)$ for all elements $\beta \notin S$. Then, by Lemma 1 , we have $b \geq 2 n$. Since

$$
b n-1+b \sqrt{m}=\epsilon^{2}+(b-2 n) \epsilon \in S
$$


we also have $a \leq b n-1$. Then,

$$
\alpha \epsilon^{-1}=(a+b \sqrt{m})(n-\sqrt{m})=a n-b m+(b n-a) \sqrt{m} .
$$

So

$$
\begin{aligned}
\operatorname{tr}_{K / \mathbb{Q}}\left(\alpha \epsilon^{-1}\right) & =2(a n-b m) \leq 2\left(n(b n-1)-b\left(n^{2}-1\right)\right) \\
& =2(b-n)<2 a=\operatorname{tr}_{K / \mathbb{Q}}(\alpha) .
\end{aligned}
$$

So $\alpha \epsilon^{-1} \in S$. Thus $\alpha \in S$. Contradiction.

Lemma 3. For $l \geq 2, \epsilon^{l}=-1+b_{1} \epsilon+b_{2} \epsilon^{2}+\ldots+b_{l-1} \epsilon^{l-1}$ where $b_{1} \geq 2 n-1$ and $b_{2}, \ldots, b_{l-1} \geq 2 n-2$.

Proof. We use induction on $l$. As $\epsilon^{2}=2 n \epsilon-1$, the assertion holds for $l=2$. If this Lemma is true for $l=s \geq 2$,

$$
\begin{gathered}
\epsilon^{s+1}=\epsilon \epsilon^{s}=\epsilon\left(-1+b_{1} \epsilon+b_{2} \epsilon^{2}+\ldots+b_{s-1} \epsilon^{s-1}\right) \\
=-\epsilon+\epsilon^{2}+\left(b_{1}-1\right) \epsilon^{2}+b_{2} \epsilon^{2}+\ldots+b_{s-1} \epsilon^{s} \\
=-1+(2 n-1) \epsilon+\left(b_{1}-1\right) \epsilon^{2}+b_{2} \epsilon^{2}+\ldots+b_{s-1} \epsilon^{s} .
\end{gathered}
$$

This proves the Lemma.

Lemma 4. If $\alpha \in \mathcal{O}_{K}^{+}, \alpha=p \epsilon^{k}+q \epsilon^{k+1}$ for some $p, q \in \mathbb{N}$ and $k \in \mathbb{Z}$.

Proof. By Lemma 2, $\alpha=a_{k} \epsilon^{k}+\ldots+a_{k+l} \epsilon^{k+l}$ with $a_{k}, \ldots, a_{k+l} \geq 0$.

If $l \geq 2$ and $a_{k+l} \leq a_{k}$,

$$
\begin{gathered}
\alpha=a_{k} \epsilon^{k}+\ldots+a_{k+l-1} \epsilon^{k+l-1}+a_{k+l} \epsilon^{k}\left(-1+b_{1} \epsilon+\ldots+b_{l-1} \epsilon^{l-1}\right) \\
=\left(a_{k}-a_{k+l}\right) \epsilon^{k}+\left(a_{k+1}+a_{k+l} b_{1}\right) \epsilon^{k+1}+\ldots+\left(a_{k+l-1}+a_{k+l} b_{l-1}\right) \epsilon^{k+l-1} .
\end{gathered}
$$

If $l \geq 2$ and $a_{k+l} \geq a_{k}$,

$$
\begin{gathered}
\alpha=a_{k} \epsilon^{k}+\ldots+a_{k+l-1} \epsilon^{k+l-1}+\left(a_{k+l}-a_{k}\right) \epsilon^{k+l}+a_{k} \epsilon^{k}\left(-1+b_{1} \epsilon+\ldots+b_{l-1} \epsilon^{l-1}\right) \\
=\left(a_{k}+a_{k+l} b_{1}\right) \epsilon^{k+1}+\ldots+\left(a_{k}+a_{k+l} b_{l-1}\right) \epsilon^{k+l-1}+\left(a_{k+l}-a_{k}\right) \epsilon^{k+l}
\end{gathered}
$$

Repeating the same process, we can obtain the desired expression of $\alpha$.

Proof of Theorem 1. If $\alpha \in \mathcal{O}_{K}^{+}$, by Lemma $4, \alpha=a \epsilon^{k}+b \epsilon^{k+1}$ for some $a, b \in \mathbb{N}$ and $k \in \mathbb{Z}$. If $k$ is even, by Lagrange's four square theorem, $a \epsilon^{k}$ is represented by $x_{1}^{2}+x_{2}^{2}+x_{3}^{2}+x_{4}^{2}$ and $b \epsilon^{k+1}$ is represented by $\epsilon x_{5}^{2}+\epsilon x_{6}^{2}+\epsilon x_{7}^{2}+\epsilon x_{8}^{2}$. So $f$ represents $\alpha$. Similarly $f$ represents $\alpha$ for odd $k$. Thus $f$ is universal integral over $K$. 
Lemma 5. There are infinitely many square free integers of the form $n^{2}-1$.

Proof. If $n$ is even, $n^{2}-1$ is square free if and only if both $n+1$ and $n-1$ are square free. It is known that [4] the number of positive square free integers which do not exceed $x$ is $\frac{6 x}{\pi^{2}}+O(\sqrt{x})$. So the number of positive integer $n$ such that $n \leq x$ and both $n+1$ and $n-1$ are square free is larger than

$$
\left(\frac{6 x}{\pi^{2}}+O(\sqrt{x})\right)+\left(\frac{6 x}{\pi^{2}}+O(\sqrt{x})\right)-x=\frac{12-\pi^{2}}{\pi^{2}} x+O(\sqrt{x})
$$

Since $\frac{12-\pi^{2}}{\pi^{2}}>0$, there are infinitely many $n$ such that $n \leq x$ and $n^{2}-1$ is square free.

Theorem 2. There are infinitely many real quadratic fields that admit octonary universal forms.

Proof. This is an immediate consequence of Theorem 1 and Lemma 5.

\section{Acknowledgement}

The content of this paper is a part of author's thesis. The author wishes to represent his greatest thanks to his advisor Prof. M.-H. Kim of Seoul National University for his kind advice and careful revision of manuscript.

\section{References}

[1] Chan, W. K., Kim, M-H., Raghavan,S., Ternary Universal Quadratic Forms over Real Quadratic Fields, Japanese J. Math. 22 (1996), 263-273.

[2] Dixon, L. E., Quaternary Quadratic Forms Representing All integers, Amer. J. Math. 49 (1927), 39-56.

[3] Götzky, F., Über eine Zahlentheoretische Anwendung von Modulfunktionen einer Veränderlichen, Math. Ann. 100 (1928), 411-437.

[4] Hardy, G. H., An introduction to the theory of numbers, fifth edition, Oxford, 1979.

[5] Kim, B. M., Finiteness of Real Quadratic Fields which admit a Positive Integral Diagonal Septanary Universal Forms, preprint.

[6] Maass, H., Über die Darstellung total positiver des Körpers $R(\sqrt{5})$ als Summe von drei Quadraten, Abh. Math. Sem. Hamburg 14 (1941), 185-191.

[7] O'Meara, O. T., Introduction to quadratic forms, Springer Verlag, 1973.

[8] Ramanujan, S., On the Expression of a Number in the Form $a x^{2}+b y^{2}+c z^{2}+d w^{2}$, Proc. Cambridge Phil. Soc. 19 (1917), 11-21.

[9] Siegel, C. L., Sums of $m$-th Powers of Algebraic Integers, Ann. Math. 46 (1945), 313-339.

[10] Willerding, M. F., Determination of all classes of positive quaternary quadratic forms which represent all (positive) integers, Bull. Amer. Math. Soc. 54, 334-337. 
Byeong Moon Kim

Department of Mathematics

College of Natural Science

Kangnung National University

123 Chibyon-Dong Kangnung

Kangwon-do 210-702

Korea

e-mail: kbm@knusun.kangnung.ac.kr

(Received: November 2, 1998) 